\title{
Profiling of skeletal muscle tissue for long non-coding RNAs related to muscle metabolism in the QingYu pig at the growth inflection point
}

\author{
Jia Luo ${ }^{1,2, a}$, Linyuan Shen ${ }^{1,3, a}$, Mailin Gan ${ }^{1,3}$, Anan Jiang ${ }^{1,3}$, Lei Chen ${ }^{1,3}$, Jideng Ma ${ }^{1,3}$, \\ Long Jin ${ }^{1,3}$, Yihui Liu ${ }^{4}$, Guoqing Tang ${ }^{1,3}$, Yanzhi Jiang ${ }^{5}$, Mingzhou $\mathrm{Li}^{1,3}$, Xuewei $\mathrm{Li}^{1,3}$, \\ Shunhua Zhang ${ }^{1,3, *}$, and Li Zhu ${ }^{1,3, *}$
}

\section{* Corresponding Authors: \\ Shunhua Zhang \\ Tel: +86-18683535030 \\ E-mail: zhangsh1919@163.com \\ Li Zhu \\ Tel: +86-13982083385 \\ E-mail: zhuli7508@163.com \\ ${ }^{1}$ College of Animal Science and Technology, Sichuan Agricultural University, Chengdu 611130, China \\ ${ }^{2}$ College of Animal Science and Technology, \\ Southwest University, Chongqing 400715, China \\ ${ }^{3}$ Farm Animal Genetic Resource Exploration and \\ Innovation Key Laboratory of Sichuan, Chengdu \\ 611130, China \\ ${ }^{4}$ Sichuan Province General Station of Animal Husbandry, Chengdu 610041, China \\ ${ }^{5}$ College of Life Science, Sichuan Agricultural \\ University, Ya'an 625014, China \\ a These two authors contributed equally to this work.}

ORCID

Jia Luo

https://orcid.org/0000-0002-1582-5174

Linyuan Shen

https://orcid.org/0000-0001-6072-3268

Mailin Gan

https://orcid.org/0000-0001-9900-3559

Anan Jiang

https://orcid.org/0000-0002-5103-4361

Lei Chen

https://orcid.org/0000-0003-4375-426X Jideng Ma

https://orcid.org/0000-0001-8981-3339

Long Jin

https://orcid.org/0000-0003-0661-4989

Yihui Liu

https://orcid.org/0000-0001-9410-1280

Guoqing Tang

https://orcid.org/0000-0002-6920-5050

Yanzhi Jiang

https://orcid.org/0000-0002-9568-557X

Mingzhou Li

https://orcid.org/0000-0001-8681-7684

Xuewei Li

https://orcid.org/0000-0003-4560-5137

Shunhua Zhang

https://orcid.org/0000-0003-0569-0146

Li Zhu

https://orcid.org/0000-0001-7342-0880

Submitted Jun 22, 2020; Revised Sept 9, 2020; Accepted Sept 28, 20
Objective: Investigation of muscle growth at different developmental stages is an appropriate strategy for studying the mechanisms underlying muscle development and differences in phenotypes. In particular, the muscle development mechanisms and the difference between the fastest and slowest growth rates.

Methods: In this study, we used a growth curve model to fit the growth inflection point (IP) of QingYu pigs and compared differences in the long non-coding RNA (lncRNA) transcriptome of muscle both at the growth IP and plateau phase (PP).

Results: The growth curve of the QingYu pig had a good fit $\left(\mathrm{R}^{2}=0.974\right)$ relative to a typical S-curve and reached the IP at day 177.96. At the PP, marbling, intramuscular fat, and monounsaturated fatty acids had increased significantly and the percentage of lean muscle and polyunsaturated fatty acids had decreased. A total of 1,199 mRNAs and 62 lncRNAs were differentially expressed at the IP compared with the PP. Additional to gene ontology and Kyoto encyclopedia of genes and genomes pathway analyses, these differentially expressed protein coding genes were principally related to muscle growth and lipid metabolism. Conclusion: Our results suggest that the identified differentially expressed lncRNAs, could play roles in muscle growth, fat deposition and regulation of fatty acid composition at the IP and PP.

Keywords: lncRNA; Growth Curve; Inflection Point; Muscle; Metabolism; QingYu Pig

\section{INTRODUCTION}

Myogenesis is a complex process of the formation of muscle fibers. The formation of new multinucleated muscle fibers from mononucleated precursor cells termed myoblasts, is exclusively a prenatal process that determines the characteristics of muscles, such as numbers of fiber, possibly related to muscle strength and function [1]. In pigs, muscle growth is predominantly determined during prenatal skeletal muscle development [2]. Pigs have been genetically selected for efficient muscle growth over many previous decades, which means they are an interesting model for the study of myogenesis [3]. The classical growth development model of the pig is a sigmoidal curve. After birth, the growth rate increasing slowly until the maximum growth rate is reached at the inflection point (IP), then decreases asymptotically, reducing to a minimum at the plateau [4]. Improving growth rate and muscularity has been the primary focus of pig breeders during past decades. Therefore, muscle phenotypic difference between the maximum growth rate point (at the IP) and the minimum growth rate point (at the plateau phase, PP) represent a poten- 
tially good model for studying the molecular mechanism of muscle development. Study of the underlying complex molecular mechanisms of muscle development is beneficial for the genetic improvement of meat quality and lean meat percentage. Moreover, since pigs have similar physiological, pathological and genomic characteristics to humans, understanding the molecular mechanisms of myogenesis has important implications for understanding muscle development and muscle regeneration.

The majority of long non-coding RNAs (lncRNAs) are expressed at particular stages of biological development in a specific manner for different cells or tissues. Emerging research has shown that lncRNAs participate in the development of skeletal muscle [5], lipid deposition and adipogenesis [6]. To identify genes that affect muscle growth rates, various studies have focused on muscle transcriptome diversity at different developmental stages in the pig [7]. So far, thousands of lncRNAs have detected related to muscle growth and development. For example, Zhao et al [8] identified more than 570 lncRNAs by systematically analyzing lncRNA expression in skeletal muscle at different times. However, no studies were focused on IncRNAs at the extremes of growth rate defined by a growth curve, such as the growth IP or the PP.

The QingYu pig is an indigenous breed from Southwestern China that is medium-sized and famous for its excellent meat quality attributes. Furthermore, this breed is classified as a meat and fat dual-purpose breed. To ascertain the growth characteristics of the QingYu pig, its growth curve was modeled. And then the maximum grow rate occurring at IP was estimated. A comprehensive survey of global gene expression changes in the longissimus dorsi muscle was subsequently conducted at IP and PP. The present study was to identify differentially expressed lncRNAs linked to phenotypic differentiation (e.g. growth rate, meat quality) between the maximum growth rate point (at IP) and the minimum growth rate point (at PP). This study will serve as a valuable resource to study muscle development and it is beneficial to the breeding of animal growth and development.

\section{MATERIALS AND METHODS}

All animal procedures were conducted in accordance with institutional guidelines for the care and use of laboratory animals and were approved by the Animal Care and Ethics Committee of Sichuan Agricultural University, Sichuan, China, under Permit number DKY-B20161705.

\section{Animal and sample preparation}

Animal experiments were organized by Sichuan Agricultural University and conducted at BaShan Animal Husbandry Limited Technology Co., Ltd. Growth development data of
126 captive QingYu pigs (56 males and 60 females) at 18 time points were collected from birth to $400 \mathrm{~d}$ in an unselected, random mating population. Body weight and other measurements were collected from two batches of the pigs. The two batches have uniform environmental conditions except for a difference in age of birth of 20 days. Growth weight was measured then a growth curve fitted using three non-linear models. All pigs were housed in individual pens $\left(2 \mathrm{~m}^{2}\right)$ located in the same room and fed twice daily using the same diet, with ad libitum access to water, using nipple drinkers. Experimental diets, based on corn and soybean meal, were formulated with crude protein, trace mineral and vitamin concentrations that met or exceeded the National Research Council (NRC, 1998) recommendations for different growth phases. At slaughter age (IP, 178 days old and PP, 395 days old), mean body weights of the pigs were $66.98 \pm 6.6 \mathrm{~kg}$ and $145.9 \pm 9.5 \mathrm{~kg}$, respectively. After slaughter, the longissimus dorsi muscle was sampled into $2 \mathrm{~mL}$ tubes within $30 \mathrm{~min}$, immediately frozen in liquid nitrogen then transferred to a freezer at $-80^{\circ} \mathrm{C}$ for long-term preservation and additional total RNA extraction, if required.

\section{Growth curve models}

There are three nonlinear models involved in our study. The first is the logistic growth curve model, the equation is $\mathrm{W}_{\mathrm{t}}=$ $\mathrm{A} /\left(1+\mathrm{B} e^{-k t}\right)$. The second is the Gompertz growth curve model, defined by the equation $\mathrm{W}_{\mathrm{t}}=\mathrm{A} e^{-B e^{-k t}}$. The third is the Von Bertalanffy growth curve model, which is defined by the equation $\mathrm{W}_{\mathrm{t}}=\mathrm{A} \times\left(1-\mathrm{B} e^{-k t}\right)^{3}$. When fitting a curve by these models, $\ln \mathrm{B} / \mathrm{k}, \ln \mathrm{B} / \mathrm{k}$, and $\ln \mathrm{B} / \mathrm{k}$ represent the inflection age; $\mathrm{A} / 2, \mathrm{~A} / \mathrm{e}$, and $8 \mathrm{~A} / 27$ represent the inflection weight; $\mathrm{kw} / 2$, $\mathrm{kw}$, and $3 \mathrm{kw} / 2$ represent the maximum daily gain. In the above equations, $\mathrm{W}_{\mathrm{t}}$ represents the time point where the weight was recorded, A represents the maximum size, $\mathrm{k}$ may be interpreted as the inherent relative growth rate at the start, and $\mathrm{B}$ is the growth curve line constant.

Goodness-of-fit $\left(R^{2}\right)$ was used to judge the merits of the fitting model, the equation is $\mathrm{R}^{2}=1-\frac{\mathrm{RSE}}{\mathrm{RST}}$, where $\mathrm{R}^{2}$ represents the goodness-of-fit, RSE represents the residual sum of squares, and RST represents the sum of squares of deviations.

\section{Carcass and meat quality measurements}

After evisceration, the left half carcass was weighed, and the dressing percentage calculated. The right half of the carcass was used to assess morphometric parameters. Specifically, carcass length was measured using a flexible tape. The loin eye area was also measured at the level of the 6th to 7 th rib. The dorsal fat thickness was measured with a flexible tape at the level of the first rib, the last rib, and in the region where the dorsal fat was the thickest. The mean of these three mea- 
surements was used for the comparison of dorsal fat values.

Meat quality traits including muscle $\mathrm{pH}$, meat color, drip loss, cooking loss and Warner-Bratzler shear force (WBS) were measured at both $45 \mathrm{~min}$ post-mortem. Marbling scores were evaluated $24 \mathrm{~h}$ post-mortem using a published visual standard (NPPC, 1991). Muscle $\mathrm{pH}$ was measured at approximately $1 \mathrm{~cm}$ below the cutting surface of longissimus dorsi (3rd to 4th rib) using a pH-star meter (SFK Inc., Berlin, Germany). Meat lightness (CIE L*) was also objectively measured in the cutting surface of longissimus dorsi between 5 th and 6th rib, using the Model CR-300 Minolta Chroma Meter (Minolta, Ramsey, NJ, USA) fitted with a 50-mm-diameter aperture, using a D65 illuminant. Drip loss was determined by weighing sliced meat stored at $4^{\circ} \mathrm{C}$ after $24 \mathrm{~h}$ post-mortem, and calculated as a percentage original weight of the sliced meat. To determine cooking loss, a $2.5 \mathrm{~cm}$ thick (approximately $100 \mathrm{~g}$ ) section of loin sample was cooked to an internal temperature of $70^{\circ} \mathrm{C}$ in a steamer. Cooking loss was determined by calculating the weight difference between cooked and uncooked samples. The WBS was determined using a Texture Analyzer (TA.XT. Plus, Stable Micro Systems, Godalming, UK) equipped with a Warner-Bratzler shearing device. For determination of intramuscular fat content, 50 g samples of loin meat were collected, and the IMF was analyzed using the Soxhlet method.

\section{Fatty acid composition}

For fatty acid composition, lipids were extracted with chloroform and methanol. For lipid hydrolysis, an aliquot of lipid extract $\left(30 \mathrm{mg}\right.$ ) and $3 \mathrm{~mL}$ of $4 \% \mathrm{H}_{2} \mathrm{SO}_{4}$ in methanol were combined in a screw-capped test tube. The test tube was placed in boiling water $\left(100^{\circ} \mathrm{C}\right)$ for $20 \mathrm{~min}$ and subsequently cooled at room temperature. The resulting free fatty acids were methylated with $1 \mathrm{~mL}$ of $14 \%$ boron trifluoride in methanol at room temperature for $30 \mathrm{~min}$. Water $(1 \mathrm{~mL})$ and hexane $(5 \mathrm{~mL})$ were added. Samples were vortexed and centrifuged at $500 \times \mathrm{g}$ for $10 \mathrm{~min}$. The upper organic solvent layer was used to determine fatty acids composition. Fatty acid methyl esters were analyzed on a gas chromatograph (Agilent Technologies 6890 N, Santa Clara, CA, USA) equipped with an on-column injection port and flame-ionization detector. A Silica capillary column (Omegawax 320, $30 \mathrm{~m} \times 0.32 \mathrm{~mm} \times 0.25 \mu \mathrm{m}$ film; Supelco, Bellefonte, PA, USA) was used for the separation of the fatty acid methyl esters. The gas chromatography oven temperature was $140^{\circ} \mathrm{C}$, and increased at a rate of $2^{\circ} \mathrm{C} / \mathrm{min}$ to a final temperature of $230^{\circ} \mathrm{C}$. The temperatures of injector port and detector temperatures were set at $240^{\circ} \mathrm{C}$ and $250^{\circ} \mathrm{C}$, respectively. Fatty acid methyl ester $(1 \mu \mathrm{L})$ was injected onto the split injection port (100:1 split ratio). The flow rate for He carrier gas was $50 \mathrm{~mL} / \mathrm{min}$. Each fatty acid was identified by its retention time.

\section{Isolation of total RNA and quality control}

Longissimus dorsi muscles at IP and PP from three female pigs were harvested for total RNA isolation. A mirVana RNA isolation kit (\#AM1561, Ambion, Austin, TX, USA) was used to isolate total RNA, in accordance with the manufacturer's instructions. Isolated total RNA from each sample was preserved at $-80^{\circ} \mathrm{C}$. A NanoDrop 2000 spectrophotometer (Thermo Scientific, Waltham, MA, USA) was used to determine RNA concentration from the optical density (OD)-260 nm/OD-280 nm absorption ratio, which was controlled in the range of 1.9 to 2.1. A Bioanalyzer 2100 (Agilent, USA) was used to evaluate the quality of total RNA (RNA integrity number $\geq 7$ and $28 \mathrm{~S} / 18 \mathrm{~S} \geq 0.7$ ). RNA integrity number was larger than 8.0 in all samples. RNase-free DNase I (Ambion Inc., USA) was used to eliminate potential genomic DNA contamination.

\section{RNA-seq, data processing and gene annotation}

Total RNA was extracted from longissimus dorsi of IP and PP using TRIZOL (Invitrogen, Carlsbad, CA, USA), and further purified with RNeasy column (Qiagen, Valencia, CA, USA) according to the manufacturer's protocol. Three biological replicates in each group were used to construct transcriptome Library. Approximately $3 \mu \mathrm{g}$ of total RNA from each sample were used for the construction of cDNA libraries (including IP1, IP2 and IP3; PP1, PP2, and PP3), in accordance with the Illumina TruSeq RNA sample preparation guide. The process included: i) separation, enrichment and purification of mRNA using oligo (dT) magnetic beads; ii) enzymatic fragmentation of RNA; iii) synthesis of cDNA; iv) sequencing adapter ligation; and v) polymerase chain reaction (PCR) amplification. An Agilent DNA 1000 kit was used to determine the size and purity of cDNA libraries on an Agilent 2100 Bioanalyzer (Agilent technologies, USA). An ABI StepOnePlus qRT-PCR system was used to accurately determine the effective concentration of cDNA libraries $(>2$ $\mathrm{nmol} / \mathrm{L}$ ), thus ensuring their quality. An Illumina HiSeq 2500 platform was used for paired-end sequencing of cDNA libraries from which raw reads were obtained. The raw reads were transferred from original image data by base calling. Quality control and reads statistics were determined by FASTQC v0.11.2. After discarding the reads containing adapter, reads containing over $10 \%$ poly-Ns, and reads of low quality ( $>50 \%$ of bases with Phred scores $<5)$, the remaining clean reads were aligned to the reference $S$. scrofa genome (10.2) using TopHat v2.0.9, package, and parameters were set as default.

The RNA sequencing data were uploaded to NCBI's Gene Expression Omnibus, and can be found under Accession Number GSE139322 https://www.ncbi.nlm.nih.gov/geo/ query/acc.cgi?acc=GSE139322. 


\section{Identification of lncRNAs and mRNAs}

All the downstream analyses were based on high quality clean data. The mapped reads from each library were assembled with Cufflinks (version 2.1.1) to construct and identify mRNA transcripts. Next, the data analysis was performed by filtering the assembled novel transcripts from the different libraries to obtain putative lncRNAs following the steps in the pipeline as follows. First, transcripts that were shorter than $200 \mathrm{nt}$ in length, containing fewer than 1 exon and fewer than three reads were excluded. Next, using the coding-non-coding index (CNCI) and coding potential calculator (CPC) to evaluate the coding potential of the filtered transcripts. A transcript with a CNCI value lower than 0 and a CPC value lower than -1 was taken to be an lncRNA. The expression levels of mRNAs and lncRNAs were quantified using a TPM algorithm [9].

\section{Analysis of differentially expressed genes}

Three biological replicates were utilized in this experiment. Differentially expressed lncRNAs and mRNAs were identified based on a negative binomial distribution using the Noiseq package [10]. Differentially expressed mRNAs and lncRNAs were filtrated by Cuffdiff software with parameters of $\mathrm{p}$ value $<0.05$ and $\mid \log 2$ (fold change) $\mid \geq 1$.

\section{Neighboring genes prediction and functional analysis} of differentially expressed lncRNAs

LncRNAs can cis-regulate neighboring genes. For expression pattern analysis, a Pearson correlation coefficient (PCC) was calculated for expression values of each $\operatorname{lncRNA}$ and mRNA. The PCCs of expression levels of differentially expressed $\operatorname{lncRNAs}$ and mRNAs were calculated. $|\mathrm{PCC}|>0.8$ and $\mathrm{p}$ value $<0.05$ were the threshold according to which co-expressed lncRNA-mRNA were selected. Genes transcribed within a $100-\mathrm{kb}$ window upstream or downstream of lncRNAs were considered cis neighboring gene $s$ if the |PCC $\mid$ of lncRNA-mRNA >0.9. Gene ontology annotation and Kyoto encyclopedia of genes and genomes (KEGG) [11] pathway enrichment analysis were conducted for those neighboring genes to investigate the biological processes and signaling pathways with which the lncRNAs were principally involved and their functions.

\section{Quantitative real-time polymerase chain reaction verification}

The relative expression levels of selected genes were quantified using real-time RT-PCR analysis same as our previous research. Briefly, total RNA was extracted using Trizol reagent (Invitrogen Corp, USA), accordance with the manufacturer's instructions. Reverse transcription was performed using oligo (dT) random 6-mers primers provided in the PrimeScript RT Master Mix kit (TaKaRa, Dalian, China). Quantitative PCR was conducted using a SYBR Premix Ex Taq kit (TaKaRa, China) with using a CFX96 real-time PCR detection system (Bio-Rad, Richmond, CA, USA). All measurements included a negative control (no cDNA template) and each RNA sample was analyzed in triplicate. Relative expression levels of the target mRNAs and lncRNAs were calculated using the $2^{-\Delta \Delta} \mathrm{Ct}$ method against the endogenous control glyceraldehyde3-phosphate dehydrogenase.

\section{Statistical analysis}

All data are presented as means \pm standard deviations. The significance of comparisons between the IP and PP were calculated using a Student's t-test. Statistical significance is defined when $\mathrm{p}$ values are less than 0.05 . Benjamin-corrected modified Fisher's exact test was used to calculate the $\mathrm{p}$ values.

\section{RESULTS}

\section{Growth curve of the QingYu pig}

The growth curve and growth rates of the QingYu pig are displayed in Figure 1. The QingYu pig growth curve fitted well to a typical S-curve. The relative growth intensity of younger pigs was greater than that of older pigs, and decreasing gradually with age. As shown in Table 1 and Figure 1, all fitted lines were closed to the observed experimental values, the models able to be ranked according to their $\mathrm{R}^{2}$ values as logistic (0.974). The growth curve suggested that QingYu pigs reached their maximum growth rate at IP (at day 177.96) when the mean body weight was $65.20 \mathrm{~kg}$, then the growth rate gradually declined. Based on the growth curve of QingYu pigs, the longissimus dorsi muscle was harvested at the IP (day 178, IP) and growth plateau (day $395, \mathrm{PP}$ ) for phenotypic and transcriptome analysis in order to reveal potential molecular regulation mechanisms of

Table 1. The estimated value of growth curve and fitting degree of different models

\begin{tabular}{lccccccc}
\hline Models & $\mathbf{k}$ & $\mathbf{a}$ & $\mathbf{b}$ & $\mathbf{R}^{\mathbf{2}}$ & GTPD (d) & GTPW (kg) & TPGR (g) \\
\hline Logistic & 135.63 & 22.075 & 0.017 & 0.999 & 182.03 & 67.72 \\
Von Bertalanffy & 198.903 & 0.826 & 0.005 & 0.998 & 181.49 & 58.93 & 576.4 \\
Gompertz & 165.829 & 4.141 & 0.008 & 0.996 & 185.6 & 61.01 & 488.08 \\
\hline
\end{tabular}

$\mathrm{k}$, the inherent relative growth rate at the start; $a$, the maximum size; $b$, the growth curve line constant; $R^{2}$, the goodness-of-fit; GTPD, growth turning point day; GTPW, growth turning point weight; TPGR, the biggest growth rate. 
A

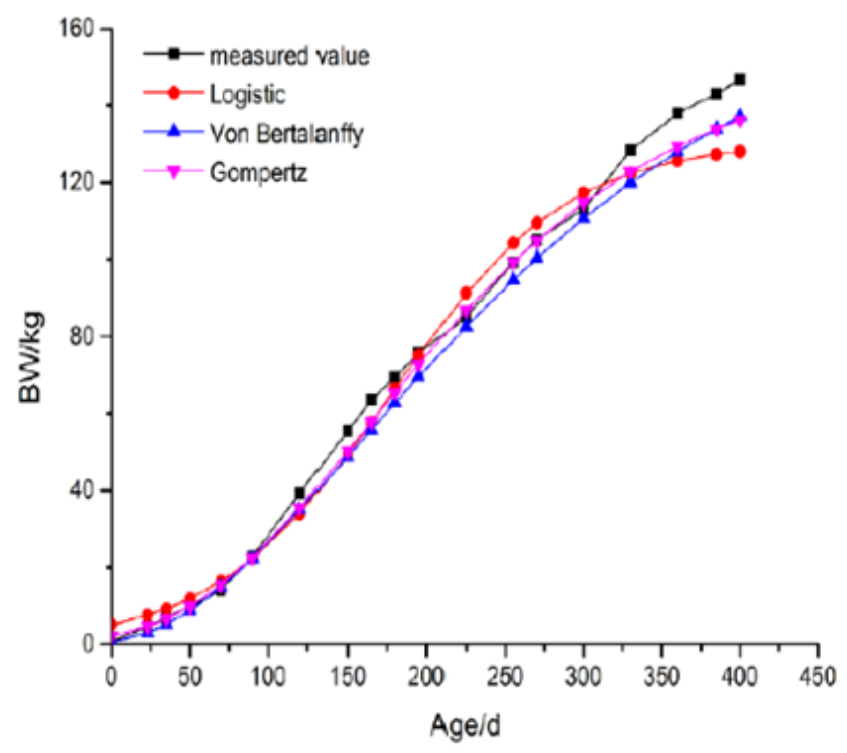

B

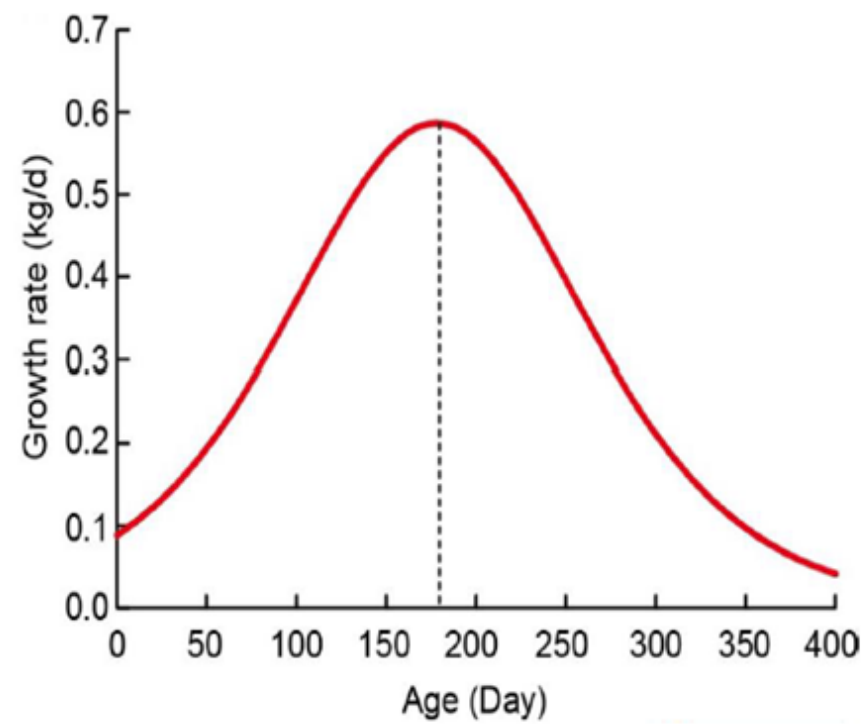

Figure 1. The fitting growth curve of QingYu pig. (A) Growth curve of QingYu pig, four curves include one measured value and three curves fitted by three different models (Logistic, Gompertz and Von Bertalanffy). (B) Growth rates of QingYu pig.

muscle growth and metabolism.

\section{Phenotypic differences of muscle at the inflection point} and plateau phase

Carcass and meat quality is influenced by the age $[12,13]$. To determine differences at different developmental stages, the carcass and meat quality characteristics of the QingYu pig at IP and PP were compared based on the growth curve of QingYu pigs (Table 2). The dressing percentage, back fat, cooking loss, marbling and intramuscular fat all increased significantly $(\mathrm{p}<0.05)$ at the PP. Fat development within muscle is not late maturing, however, marbling and intramuscular fat are known as a late maturing trait. Moreover, the expression of marbling is due to maintained fat synthesis in combination with declining muscle growth as animals get older [14]. Moreover, lean percentage, drip loss and $\mathrm{pH}$ decreased significantly $(\mathrm{p}<0.05)$. These findings demonstrate that fat deposition was enhanced in later feeding.

Fatty acids are the principal substrate and primary fuel source of lipid metabolism, also closely associated with adipocyte development [15]. Furthermore, fatty acid content is an important factor that affects the flavor of meat. Fatty acids are divided into three types according to the degree of saturation: saturated fatty acids (SFA), monounsaturated fatty acids (MUFA), and polyunsaturated fatty acids (PUFA). Cameron et al. found the longissimus dorsi muscle in Duroc pigs had higher SFA and MUFA content, with lower concentrations of PUFA than in Landrace pigs. However, the fatty acid content and composition in different growth phases is unclear.
Therefore, we analyzed the fatty acid content and composition at the IP and PP (Figure 2). Our results indicated that muscles at the PP exhibited significantly greater total fatty acid and MUFA content than at the IP $(\mathrm{p}<0.001)$. PUFA concentration at the PP was significantly lower than that of the IP $(\mathrm{p}<0.05)$. Oleic acid $(\mathrm{C} 18: 1)$ content was $110.07 \mathrm{mg} / \mathrm{g}$ at the IP vs $302.44 \mathrm{mg} / \mathrm{g}$ at the PP. In addition, both C18:2 (linoleic acid, 28.23 vs $36.65 \mathrm{mg} / \mathrm{g}$ ) and C18:3 (linolenic acid,

Table 2. Carcass, meat quality characteristics of QingYu pig at the inflection point (IP) and plateau phase (PP)

\begin{tabular}{lccc}
\hline Age/d & IP & PP & p-value \\
\hline Slaughter weight (kg) & $66.98 \pm 6.6$ & $145.9 \pm 9.5$ & $<0.001$ \\
Carcass weight $(\mathrm{kg})$ & $44.64 \pm 3.99$ & $112.97 \pm 8.55$ & $<0.001$ \\
Dressing percentage $(\%)$ & $66.86 \pm 5.03$ & $77.73 \pm 1.92$ & $<0.05$ \\
Back fat (mm) & $33.28 \pm 5.4$ & $52.29 \pm 9.03$ & $<0.05$ \\
Eye muscle $\left(\mathrm{cm}^{2}\right)$ & $17.84 \pm 3.67$ & $19.46 \pm 5.26$ & $>0.05$ \\
Lean percentage (\%) & $43.32 \pm 2.94$ & $35.54 \pm 2.00$ & $<0.05$ \\
Leg hip ratio (\%) & $25.64 \pm 0.76$ & $25.73 \pm 2.02$ & $>0.05$ \\
Marbling & $2.63 \pm 0.41$ & $2.75 \pm 0.28$ & $<0.05$ \\
Drip loss (\%) & $1.90 \pm 0.50$ & $1.75 \pm 0.28$ & $<0.05$ \\
Cooking loss (\%) & $66.03 \pm 2.29$ & $70.48 \pm 1.49$ & $<0.05$ \\
pH & $6.80 \pm 0.31$ & $6.37 \pm 0.32$ & $<0.05$ \\
Muscle color & $43.65 \pm 3.25$ & $44.43 \pm 1.30$ & $>0.05$ \\
Shear force & $6.05 \pm 2.67$ & $6.07 \pm 1.47$ & $>0.05$ \\
Intramuscular fat & $2.48 \pm 0.30$ & $6.52 \pm 2.77$ & $<0.01$ \\
\hline
\end{tabular}

The significance of comparisons between the inflection point (IP) and plateau phase (PP) were calculated using a Student's t-test. $p<0.05$ was considered statistically significant, $p<0.001$ was considered statistically extremely significant, $p>0.05$ was considered statistically no significant. 
A

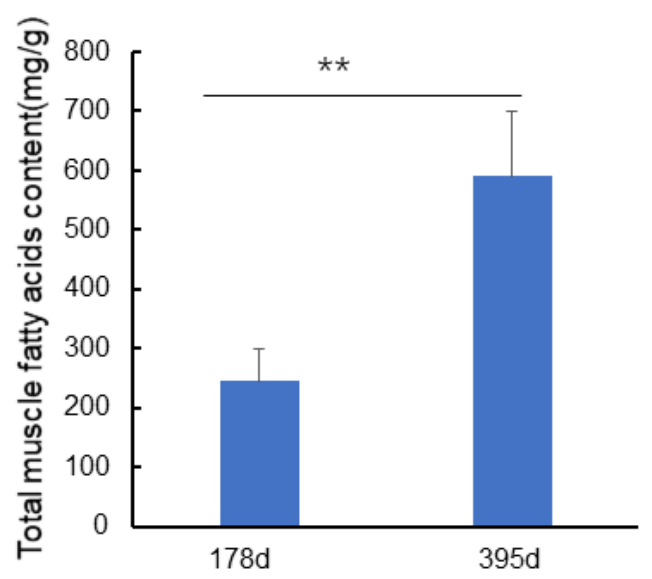

B

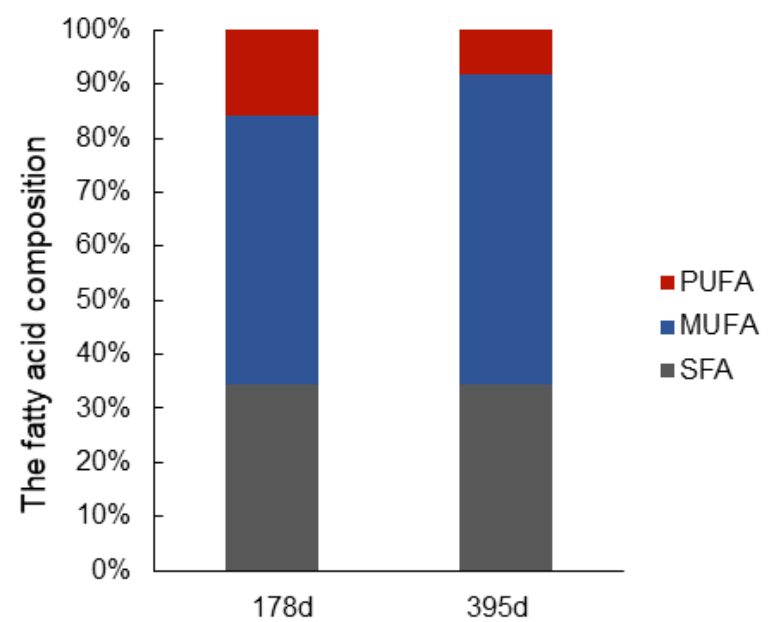

Figure 2. The fatty acid composition of QingYu pig at IP and PP, 5 pigs were used for the analysis. (A) Total muscle fatty acids content (mg/g). (B) Fatty acid composition. IP, the growth inflection point (day 178); PP, the plateau phase (day 395); PUFA, polyunsaturated fatty acid; MUFA, monounsaturated fatty acid; SFA, saturated fatty acid. ** $p<0.01$.

0.57 vs $0.69 \mathrm{mg} / \mathrm{g}$ ) content was higher at the PP.

\section{Comparison of mRNA and lncRNA characteristics}

Based on the growth curve of QingYu pigs, the longissimus dorsi muscle was harvested at the IP and PP for transcriptome analysis. To comprehensively understand the molecular regulation mechanisms of muscle growth based on the growth curve of the QingYu pig, total RNA of muscle harvested at the IP and PP was isolated and sequenced using an Illumina sequencing platform. The Q30 was not less than $91.78 \%$. After filtering, approximately $89,680,136$ of clean reads per library were typically obtained. More than $94 \%$ were mapped to the pig reference genome (Sscrofa 11.1), among them, $54.06 \%$ to $64.33 \%$ had a unique genomic position (Supplementary Table S1). A total of 22,896 transcripts were obtained, including 22,672 that were filtered according to transcriptome assembly and reconstruction. Together, these results suggest that the RNA-sequencing data were credible.

In order to further explore the characteristics of IncRNAs identified in this study, a bioinformatics analysis was performed, from which 2,192 lncRNAs and 61,379 mRNAs were identified. As shown in Supplementary Figure S1, lncRNAs and mRNAs exhibited significant differences in expression level, sequence length and exon number. FPKM values for the transcripts indicated that mRNA expression levels were relatively higher than those of IncRNAs (Supplementary Figure S1A), consistent with previous research results [16]. The majority of lncRNAs possessed one exons, significantly fewer than those of protein-coding genes. Overall, the distribution of lncRNA and mRNA lengths were consistent, and the proportion of relatively long mRNA transcripts higher than those of lncRNAs (Supplementary Figure S1B).
Mean lncRNA and mRNA length was 2,217 and 2,329 nt, respectively. mRNA transcripts were much longer than lncRNAs. The length of the majority (53.99\%) of lncRNAs was between 200 and $1,000 \mathrm{bp}$, compared to most (95.30\%) mRNAs being between $600 \mathrm{bp}$ and 3,000 bp. A comparison of mRNA and IncRNA characteristics revealed that $83.71 \%$ of the lncRNA transcripts contained 1 or 2 exons, and that $89.87 \%$ of the mRNA transcripts had $\geq 3$ exons (Supplementary Figure S1C). In addition, the number of exons in lncRNAs in muscle tissue was consistent with the number in other pig tissues.

\section{Differential expression of genes related to muscle growth and fatty acid composition}

A Venn Diagram indicated that a total of 707 mRNAs were expressed specifically at the IP and 597 mRNAs specifically at the PP of the QingYu pig (Figure 3A). A total of 1,260 differential genes, include 62 differentially expressed lncRNAs (48 up-regulated and 14 down-regulated) and 1,198 differentially expressed mRNAs (1,066 up-regulated and 132 downregulated) were identified (Figure $3 \mathrm{~B}$ ). Heatmaps of the differentially expressed genes at the IP compared with the PP suggest that they could be distinguished by clustering, reflecting the reliability of biological duplication in this study (Figure 3C).

In order to elucidate the biological functions of differentially expressed mRNAs and reveal the biological differences between muscle tissues at the IP and PP, functional enrichment analysis (including GO and KEGG Pathway analysis) was conducted on the differentially expressed mRNAs. GO enrichment analysis results demonstrated that many of the significantly enriched GO terms were closely associated with 
A

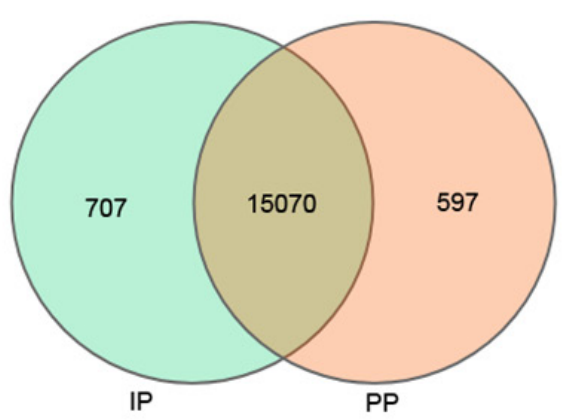

B

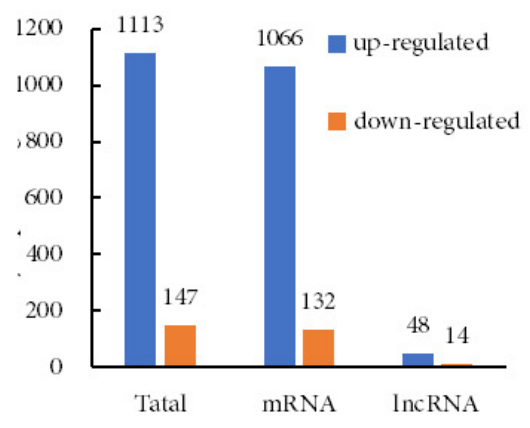

$\mathrm{C}$

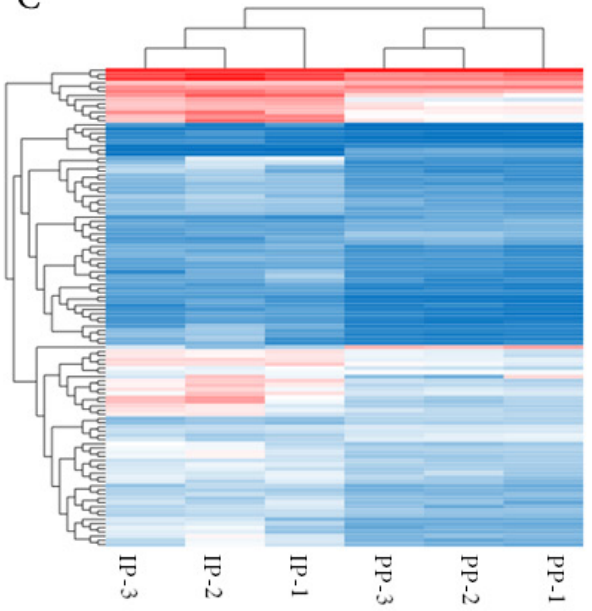

Figure 3. The expression levels of mRNA between IP and PP. (A) The Ven diagram of mRNAs that identified in two development phases. (B) The number of differentially expressed mRNA between IP and PP. (C) The hierarchical cluster analysis for the differentially expressed mRNA. IP, the growth inflection point (day 178); PP, the plateau phase (day 395).

muscle growth and development. As shown in Figure 4, in the biological process category, many genes $(\geq 10)$ were enriched in "muscle structure development", "muscle cell differentiation", "muscle system process", "muscle cell development", and "muscle tissue development". To further analyze the development-specific function of differentially expressed transcripts, a KEGG pathway enrichment analysis of differentially expressed mRNAs at the IP compared with those at the PP was conducted. As shown in Figure 5, many genes were significantly enriched in signaling pathways closely related to muscle growth and lipid metabolism, including "peroxisome proliferator activated receptor (PPAR) signaling pathway", "AMP-activated protein kinase signaling pathway", "oxidative phosphorylation", "fatty acid metabolism", and "fatty acid biosynthesis", etc.

\section{Neighboring genes and functional analysis of} differentially expressed lncRNAs

A total of 62 differentially expressed lncRNAs (48 up-regulated and 14 down-regulated) were identified. To investigate the function of lncRNAs, we predicted the potential cis targets of $\operatorname{lncRNAs}$, using $100 \mathrm{~kb}$ windows from the $\operatorname{lncRNA}$

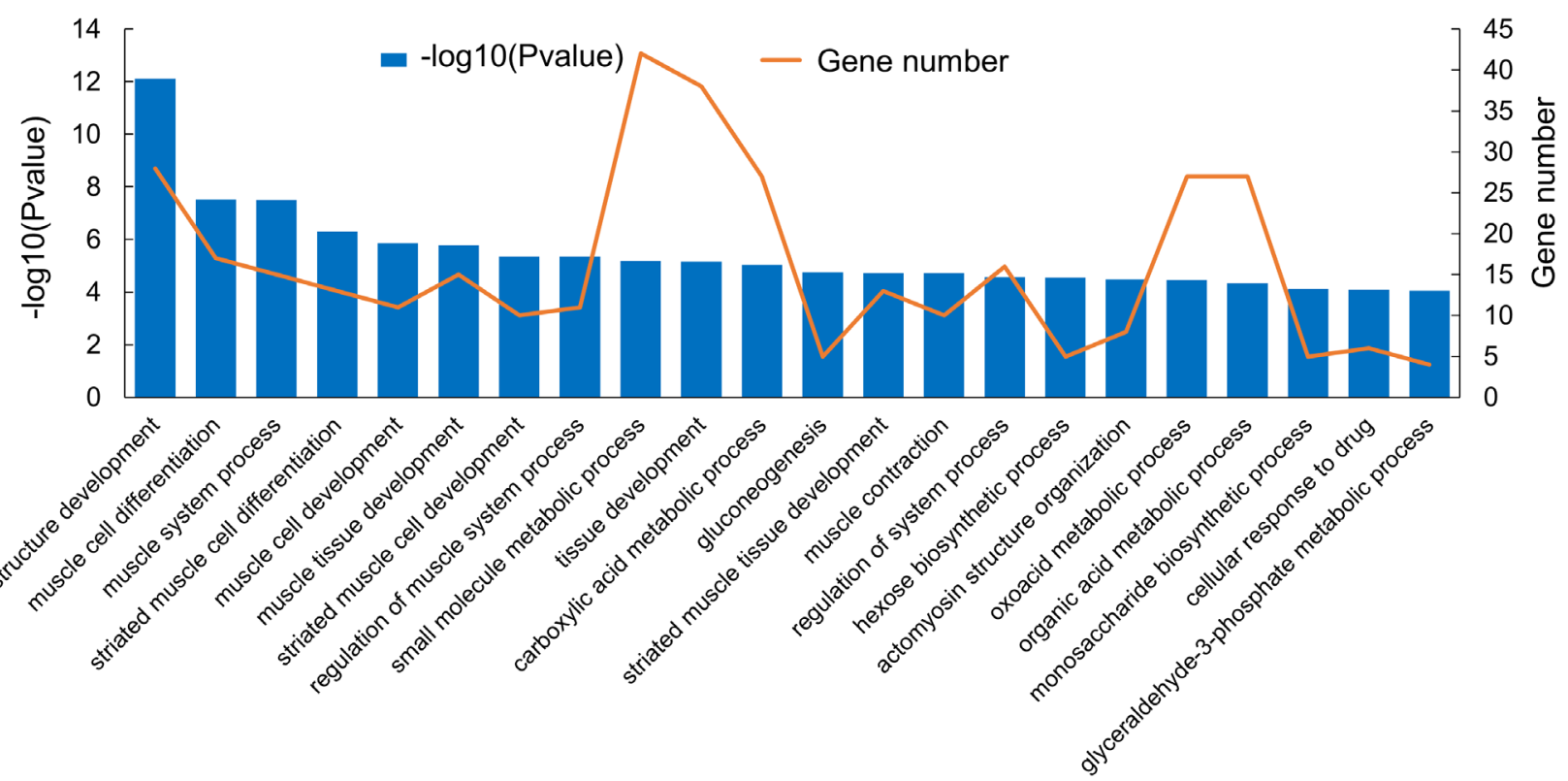

Figure 4. The functional enrichment analysis of differentially expressed mRNA in different gene ontology terms. 


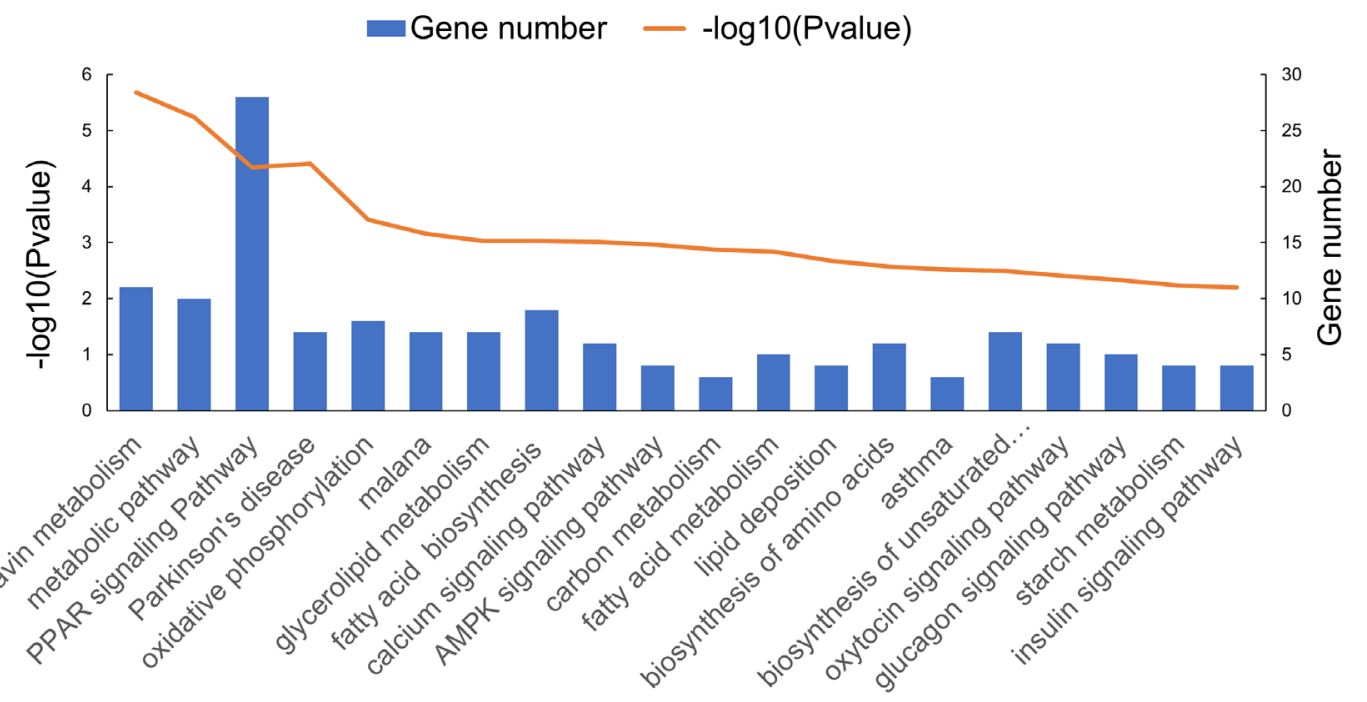

Figure 5. The functional enrichment analysis of differentially expressed mRNA in different Kyoto encyclopedia of genes and genomes pathway.

genes. Fifty six differentially expressed protein-coding genes were found close to 62 differentially-expressed lncRNA genes. GO term enrichment analysis of differentially expressed IncRNA cis-regulated predicted neighboring genes were mainly enriched in 'lipid metabolic process', 'lipid biosynthetic process', 'fatty acids biosynthetic process', 'muscle growth', and 'myoblast differentiation' (Figure 6). KEGG terms that were closely associated were: 'mitogen-activated protein kinase (MAPK) signaling pathway', 'PPAR signaling pathway', 'regulation of lipolysis in adipocytes', 'fatty acids biosynthetic process', and 'fatty acid metabolism', etc. (Figure 7). The MAPK and PPAR signaling pathways were key to the regulation of skeletal muscle development, adipocyte differentiation and lipid accumulation.

\section{Quantitative real-time polymerase chain verification}

To validate the reliability of RNA-seq results, differentially expressed genes (four lncRNAs and four mRNAs) were randomly selected for further qRT-PCR verification. qRTPCR is considered the gold standard for quantitative analysis of gene expression. The results indicated that stearoyl-CoA desaturase (SCD), parvalbumin (PVALB), regulator of calcineurin 1 (RCAN1), activating transcription factor 3 (ATF3), MSTRG.6499, and MSTRG.12897 were highly expressed

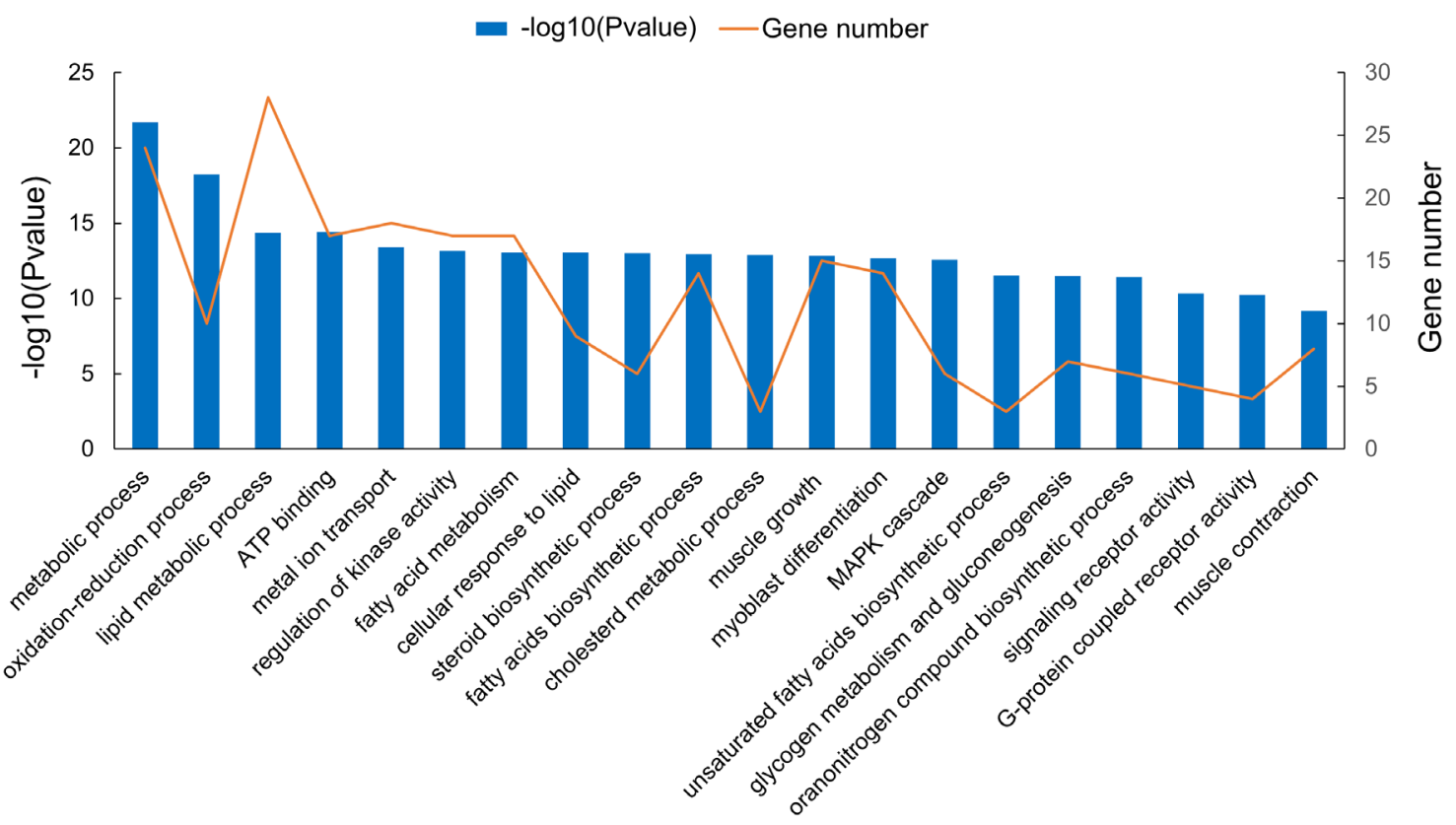

Figure 6. The functional enrichment analysis of cis-target genes by differentially expressed long non-coding RNAs. in different gene ontology terms. 


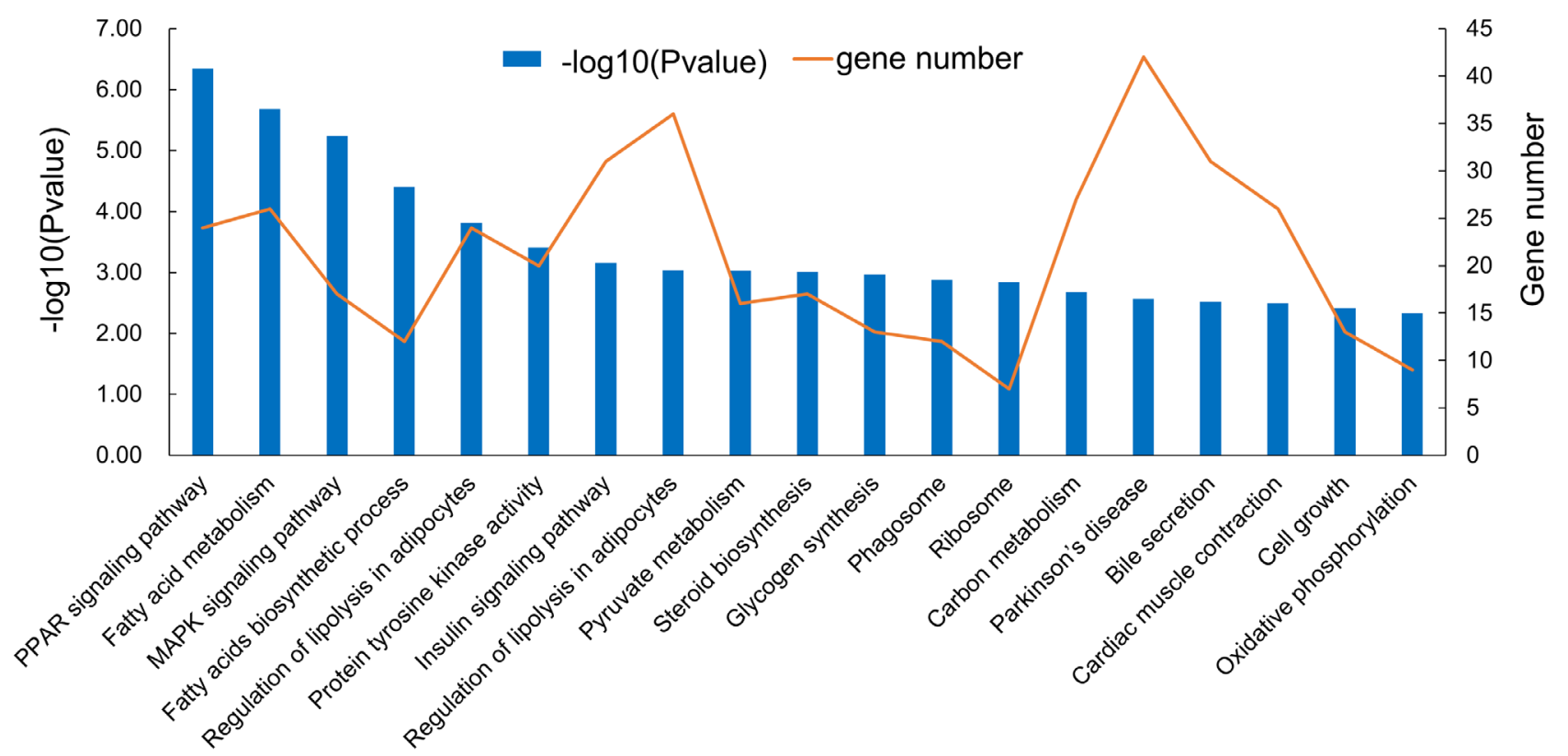

Figure 7. The functional enrichment analysis of cis-target genes by differentially expressed long non-coding RNAs in different Kyoto encyclopedia of genes and genomes pathway.

the sequencing results, verifying the reliability of the sequencing results. in IP, while MSTRG.9259, MSTRG.11973 were highly expressed in PP (Figure 8). These data were consistent with

A
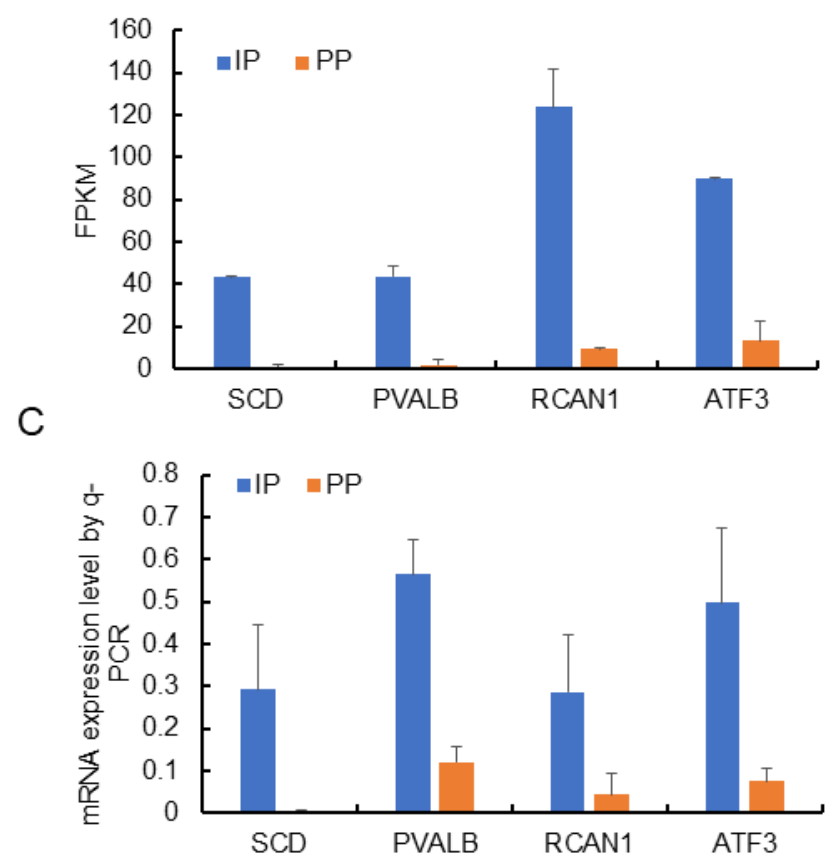

B
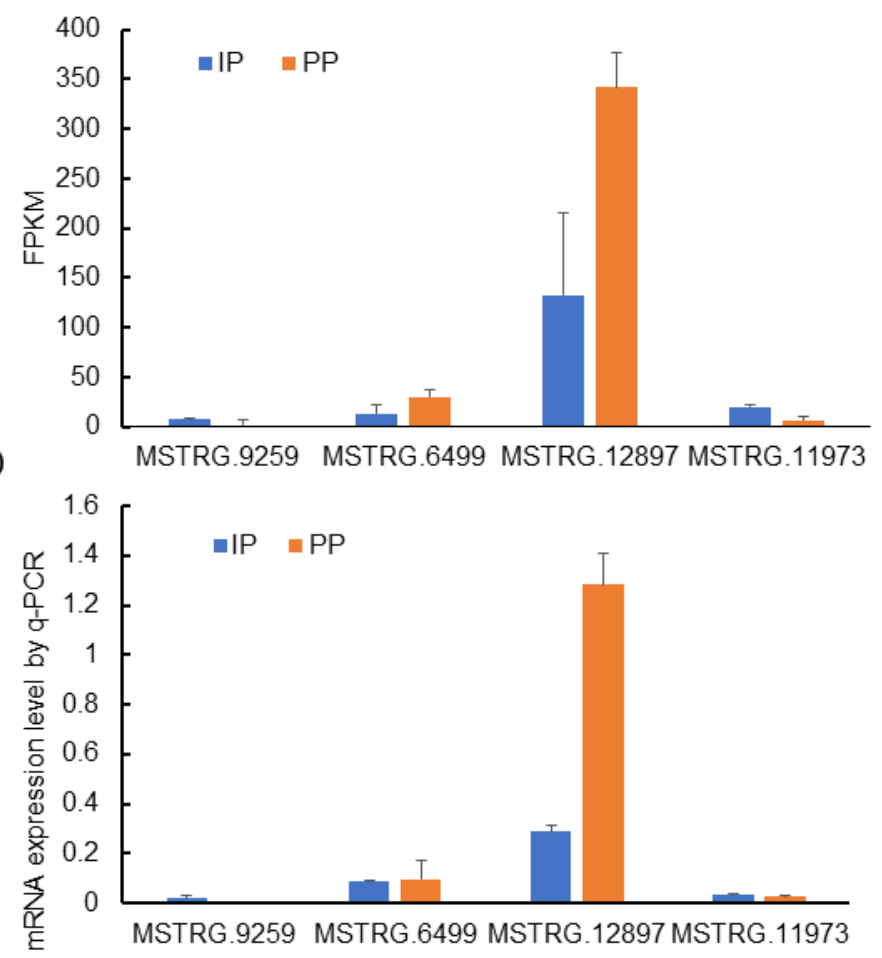

Figure 8. qRT-PCR validation of the differentially expressed genes. The differential expression of genes in muscle between IP and PP was validated by qRT-PCR, reference genes was glyceraldehyde-3-phosphate dehydrogenase. qRT-PCR, quantitative real-time polymerase chain reaction; IP, the growth inflection point (day 178); PP, the plateau phase (day 395). 


\section{DISCUSSION}

According to the growth curve, QingYu pigs reached their IP at day 177.96. QingYu pigs reached their growth IP earlier and have heavier body weight than another Chinese native breeds, such as Liangshan pigs, that reach their growth IP at day 193.40 with a mean body weight of $62.5 \mathrm{~kg}$ [17]. However, QingYu pigs reached their growth IP later and have a lower body weight than Durocs, reaching a growth IP at 163.6 days at $134.6 \mathrm{~kg}$ on average [18]. The maximum daily gain of QingYu pigs at IP was $586.82 \mathrm{~g} / \mathrm{d}$, lower than the Large White at $659.08 \mathrm{~g} / \mathrm{d}$ considerably [19]. However, similar to other reports of native Chinese pigs, the weight at the IP of the Liangshan pig is $62.5 \mathrm{~kg}$ with a maximal daily gain of $455.43 \mathrm{~g}$ and Chenghua pig is $77.73 \mathrm{~kg}$ with a maximal daily gain of $430 \mathrm{~g}$ [17]. These results suggest that QingYu pig has a lower growth rate than foreign breeds, related to the lack of long-term artificial selection for growth rate.

The marbling and intramuscular fat are increased significantly at the PP $(\mathrm{p}<0.05)$. The marbling and intramuscular fat are known as a late maturing trait and the increased marbling expression is due to the enhanced fat synthesis as animals get older. These findings demonstrate that fat deposition was enhanced in later feeding. Fatty acid composition not only affects the flavor of meat products, but is also closely related to human health. According to previous reports, PUFAs are easier to oxidize and their presence is associated with an offflavor [20]. Meat with higher PUFA content may have a worse flavor and is hard to store. Oleic acid (C18:1) content was $110.07 \mathrm{mg} / \mathrm{g}$ at the IP vs $302.44 \mathrm{mg} / \mathrm{g}$ at the PP. Reports have demonstrated that increasing C18:1 concentration pre-accelerates adipocyte differentiation, causing an increase in adipose tissues mass [21]. In addition, both C18:2 (linoleic acid, 28.23 vs $36.65 \mathrm{mg} / \mathrm{g}$ ) and C18:3 (linolenic acid, 0.57 vs $0.69 \mathrm{mg} / \mathrm{g}$ ) content was higher at the PP. Linoleic and linolenic acids are essential fatty acids in humans and the substrate for the synthesis of a variety of long-chain PUFAs such as arachidonic acid, docosapentaenoic acid and docosahexaenoic acid [22]. Their derivatives are involved in many pathways of metabolic regulation and structural formation, playing a crucial role in the growth and development of the brain [23]. These results suggest that the QingYu pig had superior muscle fatty acid composition at the PP.

Many differentially expressed genes were significantly enriched in signaling pathways closely related to muscle growth and lipid metabolism, including "muscle structure development", "muscle cell differentiation", "muscle cell development" and "PPAR signaling pathway", "AMPK signaling pathway", "oxidative phosphorylation", "fatty acid metabolism" and "fatty acid biosynthesis", etc. The AMPK signaling pathway, an important signaling system that mediates the response of cells to external stimuli, was key to regulating myoblast dif- ferentiation and fatty acid oxidation. The PPAR signaling pathway is associated with adipocyte differentiation and lipid metabolism [24]. In addition, MUFA synthesis increased with weight gain and muscle development. SCD1 is a key enzyme that converts SFAs to MUFAs and related to the biosynthesis of C18:1 and C16:1. Other important fatty acid desaturases, such as SCD2, fatty acid desaturase (FADS), were also implicated. In this study, the expression of SCD1, FADS1, and FADS2 was higher at the PP. SCD1 exhibited the biggest difference between the IP and PP. As Wang et al [25] found that genes involved in fatty acid conversion catalyzed the conversion of SFA to MUFA. However, we found SFAs were little changed between the IP and PP in QingYu pigs, whilst MUFAs increased. This finding suggests that gene expression and the biological process of fatty acid composition and metabolism in muscle may involve in other metabolic pathways.

In this research, we further predicted the potential cis targets of lncRNAs. Fifty-six differentially expressed proteincoding genes were found close to 62 differentially-expressed lncRNA genes. GO and KEGG analysis found that differentially expressed $\ln c \mathrm{RNA}$ cis-regulated predicted neighboring genes were mainly enriched in fat metabolism and muscle growth related pathways, such as 'lipid metabolic process', 'muscle growth', 'MAPK signaling pathway' and 'PPAR signaling pathway', etc. Among them, the MAPK and PPAR signaling pathways were key to the regulation of skeletal muscle development, adipocyte differentiation and lipid accumulation. Emerging research has demonstrated that lncRNAs participate in the development of skeletal muscle and fat deposition and metabolism in livestock and thousands of $\operatorname{lncRNAs}$ have been identified. For example, $55 \mathrm{lncRNAs}$ that were expressed differentially in high intramuscular fat liver compared with low intramuscular fat liver in pigs [26]. Linc-RAM enhances myogenic differentiation by interacting with MyoD, reveal the functional role of a Linc-RAM as a regulatory lncRNA required for tissues-specific chromatin remodeling and gene expression [27]. Linc-YY1 promotes myogenic differentiation and muscle regeneration through an interaction with the transcription factor YY1 [28]. Additionally, several lncRNAs and protein coding genes associated with muscle development were screened in sheep using RNAsequencing. All studies described above indicated that lncRNAs play an important role in muscle growth, fat deposition and regulation of lipid metabolism at the IP and PP.

\section{CONCLUSION}

In this study, we fitted the growth curve and evaluated the growth rate of QingYu pigs. The pigs reached IP at day 177.96. Marbling, intramuscular fat and presence of MUFA increased significantly at PP. The longissimus dorsi muscle was selected 
for transcriptome analysis at IP and PP. RNA-seq technology and bioinformatics analysis were utilized to identify differentially expressed $\operatorname{lncRNAs}$ and mRNAs of muscle tissue between the IP and PP to explore the molecular mechanisms of phenotypic differentiation. Results suggest that some of the protein-coding genes that they identified as differentially expressed are involved in processes and pathways related to muscle development, fatty acid synthesis and metabolism. This study provides a useful reference for better understanding the molecular mechanisms of animal growth and lipid metabolism based on a growth curve.

\section{CONFLICT OF INTEREST}

We certify that there is no conflict of interest with any financial organization regarding the material discussed in the manuscript.

\section{ACKNOWLEDGMENTS}

This study was supported by the Sichuan Science and Technology Program (No. 2016NYZ0050; No. SCCXTD-009), the Fundamental Research Funds for the Central Universities (SWU119028), the National Natural Science Foundation of China (No. 31530073), the earmarked fund for China Agriculture Research System (No. CARS-36-05B).

\section{REFERENCES}

1. Rehfeldt C, Fiedler I, Dietl G, Ender K. Myogenesis and postnatal skeletal muscle cell growth as influenced by selection. Livest Prod Sci 2000;66:177-88. https://doi.org/10.1016/ S0301-6226(00)00225-6

2. Picard B, Lefaucheur L, Berri C, Duclos MJ. Muscle fibre ontogenesis in farm animal species. Reprod Nutr Dev 2002; 42:415-31. https://doi.org/10.1051/rnd:2002035

3. Te Pas MFW, De Wit AAW, Priem J, et al. Transcriptome expression profiles in prenatal pigs in relation to myogenesis. J Muscle Res Cell Motil 2005;26:157-65. https://doi.org/10. 1007/s10974-005-7004-6

4. Shen L, Luo J, Du J, et al. Transcriptome analysis of Liangshan pig muscle development at the growth curve inflection point and asymptotic stages using digital gene expression profiling. PLoS One 2015;10:e0135978. https://doi.org/10.1371/journal. pone. 0135978

5. Butchart LC, Fox A, Shavlakadze T, Grounds MD. The long and short of non-coding RNAs during post-natal growth and differentiation of skeletal muscles: focus on lncRNA and miRNAs. Differentiation 2016;92:237-48. https://doi.org/10. 1016/j.diff.2016.05.003

6. Huang W, Zhang X, Li A, Xie L, Miao X. Differential regulation of mRNAs and lncRNAs related to lipid metabolism in two pig breeds. Oncotarget 2017;8:87539-53. https://doi. org/10.18632/oncotarget.20978

7. Zou C, Li J, Luo W, et al. Transcriptome analysis reveals long intergenic non-coding RNAs involved in skeletal muscle growth and development in pig. Sci Rep 2017;7:8704. https:// doi.org/10.1038/s41598-017-07998-9

8. Zhao W, Mu Y, Ma L, et al. Systematic identification and characterization of long intergenic non-coding RNAs in fetal porcine skeletal muscle development. Sci Rep 2015;5:8957. https://doi.org/10.1038/srep08957

9. Roberts A, Trapnell C, Donaghey J, Rinn JL, Pachter L. Improving RNA-Seq expression estimates by correcting for fragment bias. Genome Biol 2011;12:R22. https://doi.org/10. 1186/gb-2011-12-3-r22

10.Lee WJ, Kim M, Park HS, et al. AMPK activation increases fatty acid oxidation in skeletal muscle by activating PPARa and PGC-1. Biochem Biophys Res Commun 2006;340:2915. https://doi.org/10.1016/j.bbrc.2005.12.011

11. Kanehisa M, Sato Y, Kawashima M, Furumichi M, Tanabe M. KEGG as a reference resource for gene and protein annotation. Nucleic Acids Res 2016;44:D457-62. https:/doi.org/10.1093/ nar/gkv1070

12. Virgili R, Degni M, Schivazappa C, et al. Effect of age at slaughter on carcass traits and meat quality of Italian heavy pigs. J Anim Sci 2003;81:2448-56. https://doi.org/10.2527/2003.81102448x

13. Franco D, Carballo J, Bermñudez R, Lorenzo JM. Effect of genotype and slaughter age on carcass traits and meat quality of the Celta pig breed in extensive system. Ann Anim Sci 2016;16:259-73. https://doi.org/10.1515/aoas-2015-0056

14.Lee SH, Cho YM, Lee SH, et al. Identification of marblingrelated candidate genes in $M$. longissimus dorsi of high- and low marbled Hanwoo (Korean native cattle) steers. BMB Rep 2008;41:846-51. https://doi.org/10.5483/BMBRep.2008.41. 12.846

15. Ooi EMM, Watts GF, Ng TWK, Barrett PHR. Effect of dietary fatty acids on human lipoprotein metabolism: a comprehensive update. Nutrients 2015;7:4416-25. https://doi.org/10.3390/ nu7064416

16. Ran M, Chen B, Li Z, et al. Systematic identification of long noncoding RNAs in immature and mature porcine testes. Biol Reprod 2016;94:77. https://doi.org/10.1095/biolreprod. 115.136911

17.Luo J, Lei H, Shen L, et al. Estimation of growth curves and suitable slaughter weight of the Liangshan pig. Asian-Australas J Anim Sci 2015;28:1252-8. https://doi.org/10.5713/ajas.15. 0010

18. Strathe AB, Danfær A, Sørensen H, Kebreab E. A multilevel nonlinear mixed-effects approach to model growth in pigs. J Anim Sci 2013;88:638-49. https://doi.org/10.2527/jas.20091822

19. Dube B, Mulugeta SD, Dzama K. Genetic relationship between growth and carcass traits in Large White pigs. S Afr J Anim 
Sci 2013;43:482-92. https://doi.org/10.4314/sajas.v43i4.5

20.Meijboom PW, Stroink JBA. 2-trans,4-cis,7-cis-Decatrienal, the fishy off-flavor occurring in strongly autoxidized oils containing linolenic acid or $\omega 3,6,9$, etc., fatty acids. J Am Oil Chem Soc 1972;49:555-8. https://doi.org/10.1007/BF026 09225

21. Mersmann HJ, Ding ST. Fatty acids modulate porcine adipocyte differentiation and transcripts for transcription factors and adipocyte-characteristic proteins. J Nutr Biochem 2001;12: 101-8. https://doi.org/10.1016/S0955-2863(00)00136-4

22. Olsen RE, Henderson RJ, McAndrew BJ. The conversion of linoleic acid and linolenic acid to longer chain polyunsaturated fatty acids by Tilapia (Oreochromis) nilotica in vivo. Fish Physiol Biochem 1990;8:261-70. https://doi.org/10.1007/BF0000 4465

23.Singh M. Essential fatty acids, DHA and human brain. Indian J Pediatr 2005;72:239-42. https://doi.org/10.1007/BF0285 9265

24. Schoonjans K, Staels B, Auwerx J. The peroxisome proliferator activated receptors (PPARs) and their effects on lipid meta- bolism and adipocyte differentiation. Biochim Biophys Acta Lipids Lipid Metab 1996;1302:93-109. https://doi.org/10.1016/ 0005-2760(96)00066-5

25. Wang T, Jiang A, Guo Y, et al. Deep sequencing of the transcriptome reveals inflammatory features of porcine visceral adipose tissue. Int J Biol Sci 2013;9:550-6. https://doi.org/ 10.7150/ijbs.6257

26. Ramayo-Caldas Y, Mach N, Esteve-Codina A, et al. Liver transcriptome profile in pigs with extreme phenotypes of intramuscular fatty acid composition. BMC Genomics 2012; 13:547. https://doi.org/10.1186/1471-2164-13-547

27. Yu X, Zhang Y, Li T, et al. Long non-coding RNA Linc-RAM enhances myogenic differentiation by interacting with MyoD. Nat Commun 2017;8:14016. https://doi.org/10.1038/ncomms 14016

28.Zhou L, Sun K, Zhao Y, et al. Linc-YY1 promotes myogenic differentiation and muscle regeneration through an interaction with the transcription factor YY1. Nat Commun 2015;6: 10026. https://doi.org/10.1038/ncomms10026 